\title{
Coherent photoproduction of $J / \psi$ in nucleus-nucleus collisions in the color dipole approach
}

\author{
Agnieszka Łuszczak ${ }^{1, *}$ and Wolfgang Schäfer ${ }^{2, \dagger}$ \\ ${ }^{1}$ T. Kościuszko Cracow University of Technology, PL-30-067 Cracow, Poland \\ ${ }^{2}$ Institute of Nuclear Physics Polish Academy of Sciences, ulica Radzikowskiego 152, PL-31-342 Cracow, Poland
}

(Received 24 January 2019; revised manuscript received 7 March 2019; published 8 April 2019)

\begin{abstract}
We investigate the exclusive photoproduction of $J / \psi$ mesons in ultraperipheral heavy-ion collisions in the color dipole approach. We first test a number of dipole cross sections fitted to inclusive $F_{2}$ data against the total cross section of exclusive $J / \psi$ production on the free nucleon. We then use the color dipole formulation of Glauber-Gribov theory to calculate the diffractive amplitude on the nuclear target. The real part of the free-nucleon amplitude is taken into account consistent with the rules of Glauber theory. We compare our results to recent published and preliminary data on exclusive $J / \psi$ production in ultraperipheral lead-lead collisions at $\sqrt{s_{N N}}=2.76$ and $\sqrt{s_{N N}}=5.02 \mathrm{TeV}$. Especially at high- $\gamma A$ energies there is room for additional shadowing corrections, corresponding to triple-Pomeron terms or shadowing from large mass diffraction.
\end{abstract}

DOI: 10.1103/PhysRevC.99.044905

\section{INTRODUCTION}

Following the early theoretical work [1] and the recent measurements [2-6] (see also the review [7]), there has been recently much interest in the coherent exclusive production of vector mesons in ultraperipheral heavy-ion collisions at the Large Hadron Collider (LHC). The production takes place via the diffractive photoproduction process where one of the ions serves as a source of quasireal photons. The second ion plays the role of the hadronic target on which the diffractive photoproduction proceeds.

The production of vector mesons composed of heavy quarks, such as the $J / \psi$ is of special interest and the exclusive production of $J / \psi$ in ultraperipheral heavy-ion collisions has been investigated using a number of different theoretical approaches [8-18]. In this case, the heavy quark mass provides a hard scale which ensures a dominant contribution from short distances so that a perturbative QCD approach becomes applicable. The diffractive photoproduction then becomes a sensitive probe of the gluon structure of the target.

Much attention has been paid in the past on diffractive photo- and electroproduction of vector mesons on the proton. A large body of data has been accumulated at the German Electron Synchrotron-Hadron-Electron Ring Accelerator (DESY-HERA) facility. For a review of experimental data and of the theoretical approaches, see Ref. [19]. Here, we will

\footnotetext{
*Corresponding author: agnieszka.luszczak@desy.de

${ }^{\dagger}$ wolfgang.schafer@ifj.edu.pl
}

Published by the American Physical Society under the terms of the Creative Commons Attribution 4.0 International license. Further distribution of this work must maintain attribution to the author $(s)$ and the published article's title, journal citation, and DOI. Funded by SCOAP . use the color dipole approach, which allows us to take into account nuclear effects once the dipole cross section on a free nucleon has been fixed. To this end, we take advantage of available data on exclusive $J / \psi$ production to check a variety of dipole cross sections against them. In view of the later application to ultraperipheral heavy-ion collisions the HERA energy range is the most relevant to us.

Here, we discuss the coherent diffractive photoproduction in the same approach which we used earlier for the incoherent photoproduction of $J / \psi$ [20]. This paper is organized as follows: In Sec. II, we review the formalism and main formulas for diffractive vector meson production on nucleons and nuclei in the color dipole approach. In Sec. III, we review different parametrizations or fits of the dipole cross section. Then, in Sec. IV, we compare our predictions to available published and preliminary experimental data. We summarize our findings in Sec. V.

\section{COHERENT PHOTOPRODUCTION IN THE COLOR DIPOLE APPROACH}

\section{A. Free-nucleon target}

Let us start with a brief review of the formalism for production of a vector mesons $V$ of mass $M_{V}$ (in this paper, we concentrate on $V=J / \psi)$ at high enough energies so that the coherence length $l_{c}=2 \omega / M_{V}^{2}$ is much larger than the size of the proton $l_{c} \gg R_{N}$, where $\omega$ is the photon energy. In such a situation, the $J / \psi$ photoproduction can be described as an elastic scattering of a $c \bar{c}$ of size $r$ conserved during the interaction (see, e.g., Ref. [21]). The $\gamma \rightarrow c \bar{c}$ transition and projection of the $c \bar{c}$ pair on the bound state are encoded in the relevant light-cone wave functions, which depend also on the fraction $z$ of the photon's light-front momentum carried by the quark. The coherent diffractive amplitude on the free 
nucleon then takes a form

$$
\begin{aligned}
\mathcal{A}(\gamma N \rightarrow V N ; W, \boldsymbol{q}) \\
=2\left(i+\rho_{N}\right) \int d^{2} \boldsymbol{b} \exp [i \boldsymbol{b q}]\langle V| \exp [i(1-2 z) \boldsymbol{r} \boldsymbol{q} / 2] \\
\quad \times \Gamma_{N}(x, \boldsymbol{b}, \boldsymbol{r})|\gamma\rangle \\
=\left(i+\rho_{N}\right) \int d^{2} \boldsymbol{r} \rho_{V \leftarrow \gamma}(\boldsymbol{r}, \boldsymbol{q}) \sigma(x, \boldsymbol{r}, \boldsymbol{q}) \\
\approx\left(i+\rho_{N}\right) \int d^{2} \boldsymbol{r} \rho_{V \leftarrow \gamma}(\boldsymbol{r}, 0) \sigma(x, r) \exp \left[-B \boldsymbol{q}^{2} / 2\right] .
\end{aligned}
$$

Here, $x=M_{V}^{2} / W^{2}$, where $W$ is the $\gamma p$-c.m. system energy. Our amplitude is normalized such that the differential cross section is obtained from

$$
\begin{aligned}
& \frac{d \sigma(\gamma N \rightarrow V N ; W)}{d t} \\
& \quad=\frac{d \sigma(\gamma N \rightarrow V N ; W)}{d \boldsymbol{q}^{2}} \\
& \quad=\frac{1}{16 \pi}\left|\mathcal{A}\left(\gamma^{*} N \rightarrow V N ; W, \boldsymbol{q}\right)\right|^{2} .
\end{aligned}
$$

The overlap of light-front wave functions of the photon and the vector meson is

$$
\rho_{V \leftarrow \gamma}(\boldsymbol{r}, \boldsymbol{q})=\int_{0}^{1} d z \Psi_{V}(z, \boldsymbol{r}) \Psi_{\gamma}(z, \boldsymbol{r}) \exp [i(1-2 z) \boldsymbol{r} \boldsymbol{q} / 2] .
$$

Here, a sum over quark and antiquark helicities is implicit. The overlap depends also on photon and vector meson helicities and, in general, gives rise to nonzero helicity flip transitions. We concentrate on the helicity conserving amplitude as helicity flip transitions for heavy vector mesons are suppressed. In this case, one can also neglect the $\boldsymbol{q}$-dependent phase factor. ${ }^{1}$ All the dependence on transverse momentum transfer $\boldsymbol{q}$ is contained then in the off-forward dipole cross section for which we assume a factorized form

$$
\sigma(x, \boldsymbol{r}, \boldsymbol{q})=\sigma(x, r) \exp \left[-B \boldsymbol{q}^{2} / 2\right] .
$$

The diffraction slope $B$ depends on energy and for the explicit parametrization used, see Sec. IV A below. Explicitly, the overlap of vector meson and photon light-cone wave function, obtained from the $\gamma_{\mu}$ vertex for the $Q \bar{Q} \rightarrow V$ vertex is given by $[22,23]$

$$
\begin{aligned}
\Psi_{V}^{*}(z, \boldsymbol{r}) \Psi_{\gamma}(z, \boldsymbol{r})= & \frac{e_{Q} \sqrt{4 \pi \alpha_{\mathrm{em}}} N_{c}}{4 \pi^{2} z(1-z)}\left\{m_{Q}^{2} K_{0}\left(m_{Q} r\right) \psi(z, r)\right. \\
& \left.-\left[z^{2}+(1-z)^{2}\right] m_{Q} K_{1}\left(m_{Q} r\right) \frac{\partial \psi(z, r)}{\partial r}\right\} .
\end{aligned}
$$

For the radial wave-function $\psi(z, r)$, we choose the so-called "boosted Gaussian" wave function [22,23] as parametrized in Ref. [24] for the $J / \psi$ meson.

\footnotetext{
${ }^{1} \mathrm{~A}$ form of the phase which does not vanish at $z=1 / 2$ is common in the literature. Note that our phase is consistent with the Feynmandiagram calculations in momentum space [19].
}

The real part of the amplitude is restored from analyticity from the $x$-dependent effective intercept,

$$
\Delta_{\mathbf{I P}}=\frac{\partial \ln (\langle V|\sigma(x, r)| \gamma\rangle)}{\partial \ln (1 / x)},
$$

so that

$$
\rho_{N}=\tan \left(\frac{\pi \Delta_{\mathbf{I P}}}{2}\right) .
$$

As we discuss below, the color dipole cross section has been obtained from a fit of the total photoabsorption cross section on the nucleon, i.e., a fit to the absorptive part of the forward (Mandelstam $t=0$ ) Compton amplitude. In vector meson production, even at $\boldsymbol{\Delta}=0$, the $t=0$ limit is not reached at finite energy, and there is always a finite $t_{\min }$ due to the vector meson mass. Consequently, gluons exchanged in the amplitude carry different longitudinal momenta at small $x=$ $M_{V}^{2} / W^{2}$ we have typically, say $x_{1} \sim x, x_{2} \ll x_{1}$. In such a situation, the corresponding correction which multiplies the amplitude is the factor of Shuvaev et al. [25],

$$
R_{\text {skewed }}=\frac{2^{2 \Delta_{\mathbf{I P}}+3}}{\sqrt{\pi}} \frac{\Gamma\left(\Delta_{\mathbf{I P}}+5 / 2\right)}{\Gamma\left(\Delta_{\mathbf{I P}}+4\right)} .
$$

This correction has been studied with some rigor only for the two-gluon ladder where it accounts for the "skewedness" of gluon momentum fractions. It is to be applied only at small $x$.

\section{B. Nuclear target}

When it comes to nuclear targets, one should realize that color dipoles can be regarded as eigenstates of the interaction, and one can apply the standard rules of Glauber theory [26] for each of the eigenstates. We now require that the coherence length be much larger than the nuclear size $l_{c} \gg R_{A}$. Then, we can obtain the Glauber form of the dipole scattering amplitude,

$$
\Gamma_{A}(x, \boldsymbol{b}, \boldsymbol{r})=1-\exp \left[-\frac{1}{2} \sigma(x, r) T_{A}(\boldsymbol{b})\right] .
$$

Note that being states of fixed size color dipoles are not eigenstates of a mass operator, and the rescattering of dipoles (see Fig. 1) corresponds to the diffractive transitions $M_{i}^{2} \rightarrow M_{j}^{2}$ in the individual scatterings. The dipole rescattering therefore is a particular realization of Gribov's generalization of Glauber

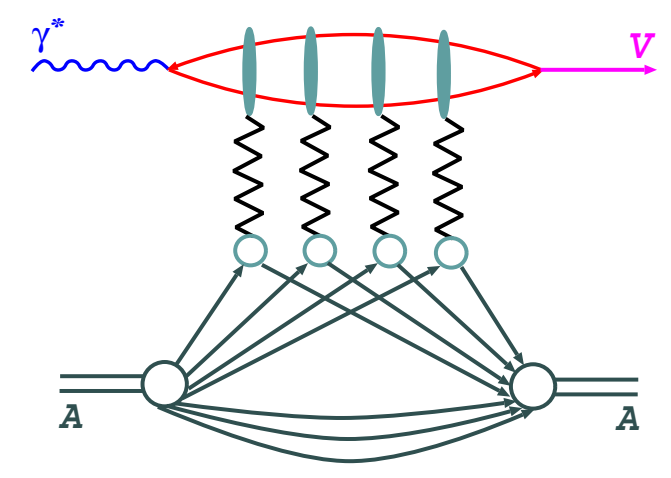

FIG. 1. Coherent photoproduction of a vector meson in which the nucleus stays in its ground state. 
theory [27]. The inelastic shadowing corrections will include masses which are different from $M_{V}^{2}$ but not much larger. That is to say that inelastic shadowing corresponding to explicit triple-Pomeron terms are not included.

The dipole amplitude of Eq. (2.9) corresponds to a rescattering of the dipole in a purely absorptive medium. The real part of the dipole-nucleon amplitude is often neglected. It induces the refractive effects [26], and instead of Eq. (2.9), we should take

$$
\begin{aligned}
\Gamma_{A}(x, \boldsymbol{b}, \boldsymbol{r})= & 1-\exp \left[-\frac{1}{2} \sigma(x, r)\left(1-i \rho_{N}\right) T_{A}(\boldsymbol{b})\right] \\
= & 1-\exp \left[-\frac{1}{2} \sigma(x, r) T_{A}(\boldsymbol{b})\right] \cos \left(\frac{1}{2} \rho_{N} \sigma(x, r) T_{A}(\boldsymbol{b})\right) \\
& +i \exp \left[-\frac{1}{2} \sigma(x, r) T_{A}(\boldsymbol{b})\right] \sin \left(\frac{1}{2} \rho_{N} \sigma(x, r) T_{A}(\boldsymbol{b})\right) .
\end{aligned}
$$

Note that the real part of the dipole amplitude $\Gamma_{A}$ contributes to the imaginary (absorptive) part of the diffractive amplitude, whereas the imaginary part of $\Gamma_{A}$ yields the real ("dispersive") part of the diffractive amplitude. We adopt the standard assumption of the nucleus being a dilute gas of uncorrelated nucleons. The optical thickness $T_{A}(\boldsymbol{b})$ is calculated from a Woods-Saxon distribution $n_{A}(\vec{r})$,

$$
T_{A}(\boldsymbol{b})=\int_{-\infty}^{\infty} d z n_{A}(\vec{r}), \quad \vec{r}=(\boldsymbol{b}, z), \quad \int d^{2} \boldsymbol{b} T_{A}(\boldsymbol{b})=A .
$$

The diffractive amplitude in $\boldsymbol{b}$ space is

$$
\mathcal{A}(\gamma A \rightarrow V A ; W, \boldsymbol{b})=2 i\left\langle V\left|\Gamma_{A}(x, \boldsymbol{b}, \boldsymbol{r})\right| \gamma\right\rangle \mathcal{F}_{A}\left(q_{z}\right) .
$$

We denote by $W$ the per-nucleon c.m. system energy in the $\gamma A$ collision. The nuclear form factor $\mathcal{F}_{A}(q)=\exp \left[-R_{\mathrm{ch}}^{2} q^{2} / 6\right]$ depends on the finite longitudinal momentum transfer $q_{z}=$ $x m_{N}$. It serves to cut off the diffractive contribution at low energies (large $x$ ) where the coherence condition is not satisfied. The total cross section for the $\gamma A \rightarrow V A$ reaction is finally obtained as

$$
\sigma(\gamma A \rightarrow V A ; W)=\frac{1}{4} \int d^{2} \boldsymbol{b}|\mathcal{A}(\gamma A \rightarrow V A ; W, \boldsymbol{b})|^{2} .
$$

\section{DIPOLE MODELS}

In the dipole picture, the deep inelastic scattering is viewed as a two-stage process; first the virtual photon fluctuates into a dipole, which consists of a quark-antiquark pair (or a $q \bar{q} g$ or $q \bar{q} g g \cdots$ system), and in the second stage, the dipole interacts with the proton. Dipole denotes a quasistable quantum-mechanical state, which has a very long lifetime $\left(\approx 1 / m_{p} x\right)$ and a size $r$, which remains unchanged during scattering. The wave-function $\Psi_{T, L}^{\gamma^{*}}\left(z, r, Q^{2}\right)$ determines the probability amplitude to find a dipole of size $r$ within a photon. This probability depends on the value of external $Q^{2}$ and the fraction of the photon momentum carried by the quarks forming the dipole $z$.

The scattering amplitude is a product of the virtual photon wave-function $\Psi$ with the dipole cross-section $\sigma(x, r)$, which determines a probability of the dipole-proton scattering. Thus, within the dipole formulation of the $\gamma^{*} p$ scattering [28],

$$
\sigma_{T, L}\left(\gamma^{*} p ; x, Q^{2}\right)=\int d^{2} \boldsymbol{r} \int_{0}^{1} d z\left|\Psi_{T, L}^{\gamma^{*}}\left(z, \boldsymbol{r}, Q^{2}\right)\right|^{2} \sigma(x, r),
$$

where $T, L$ denotes the virtual photon polarization and $\sigma_{T, L}^{\gamma^{*} p}$ denotes the total inclusive DIS cross section. It is worth remembering that besides a contribution of dipoles of sizes $r^{2} \approx 1 / Q^{2}$, the total photoabsorption also gets a scaling contribution from large dipole sizes $r^{2} \approx 1 / m_{f}^{2}$, where $m_{f}$ is a mass of the quark of flavor $f$.

Numerous models for the dipole cross section have been developed to test various aspects of the data. In the following, we will shortly review some of them, which have been obtained from fitting data on the inclusive proton structure function and which we will test against the $J / \psi$ photoproduction data before we proceed to the calculation of the nuclear observables.

We will use three different dipole models, all of which have been fitted to structure function data including charm. They differ in the treatment of the underlying QCD dynamics: the Golec-Biernat-Wüsthoff (GBW) parametrization is the most phenomenological one and rests on a geometric scaling form where the dipole cross section depends only on the variable $r^{2} Q_{s}^{2}(x)$, where $Q_{s}^{2}(x)$ is the saturation scale. The model based on the Bartels-Golec-Biernat-Kowalski parametrization is inspired by the original GBW form, but imposes DokshitzerGribov-Lipatov-Altarelli-Parisi (DGLAP) evolution on the leading-twist form of the dipole cross section. This leadingtwist cross section is then exponentiated to take account of higher-twist corrections, which are in some sense resummed to achieve the unitarization of the dipole cross section. Finally, the Iancu-Itakura-Munier (IIM) model makes use of the properties of solutions of the Balitsky-Kovchegov (BK) equation $[29,30]$.

\section{A. GBW model}

The dipole model became a popular tool in investigations of deep-inelastic scattering following the observation of Golec-Biernat and Wüsthoff $[31,32]$ that a simple ansatz for the dipole cross section was able to describe simultaneously the total inclusive and diffractive cross sections measured at HERA.

In the GBW model, the dipole-proton cross-section $\sigma_{\text {dip }}$ is given by

$$
\sigma(x, r)=\sigma_{0}\left(1-\exp \left[-\frac{Q_{s}^{2}(x) r^{2}}{4}\right]\right),
$$

where $Q_{s}^{2}(x)$ is the $x$-dependent saturation scale. It is parametrized in the form

$$
Q_{s}^{2}(x)=Q_{0}^{2} \times\left(\frac{x_{0}}{x}\right)^{\lambda} .
$$

The free fitted parameters are as follows: the cross-section normalization $\sigma_{0}$ as well as $x_{0}$ and $\lambda$. For dipole sizes which are large in comparison to the saturation radius $R \approx 1 / Q_{s}$, and the dipole cross section saturates by approaching a constant value $\sigma_{0}$, i.e., saturation damps the growth of the gluon 
density. In this model, saturation is taken into account in the eikonal approximation, and the saturation radius can be related to the gluon density in the transverse $y$ at low $x$.

The GBW model provided a good description of data from medium $Q^{2}$ values $\left(\approx 30 \mathrm{GeV}^{2}\right)$ down to low $Q^{2}$ $\left(\approx 0.1 \mathrm{GeV}^{2}\right)$. Despite its success and its appealing simplicity, the model has some shortcomings; in particular, it describes the QCD evolution by a simple $x$ dependence $\sim(1 / x)^{\lambda}$, i.e., the $Q^{2}$ dependence of the cross-section evolution is solely induced by the saturation effects. Therefore, it does not match with DGLAP QCD evolution, which is known to describe data very well from $Q^{2} \approx 4 \mathrm{GeV}^{2}$ to very large $Q^{2} \approx 10000 \mathrm{GeV}^{2}$. However, we have to remember that for the case of $J / \psi$ production the hard scale is just at the lower range of the perturbative regime: $Q^{2} \approx M_{J / \psi}^{2} / 4 \approx 2.5 \mathrm{GeV}^{2}$. Therefore one may expect that the DGLAP evolution effects are not very strong.

In this paper, we use a new fit of the GBW form of the dipole cross section obtained by Golec-Biernat and Sapeta in Ref. [33]. We take the parameters which they obtained by fitting HERA data for $Q^{2}<5 \mathrm{GeV}^{2}$, and which read $\sigma_{0}=28.18 \mathrm{mb}, \lambda=0.237$, and $x_{0}=0.31 \times 10^{-4}$ with $Q_{0}^{2}=1 \mathrm{GeV}^{2}$.

\section{B. BGK model}

The evolution ansatz of the GBW model was improved in the model proposed by Bartels, Golec-Biernat, and Kowalski (BGK) [34] by taking into account the DGLAP evolution of the gluon density in an explicit way. The model preserves the GBW eikonal approximation to saturation, and thus the dipole cross section is given by

$$
\sigma(x, r)=\sigma_{0}\left(1-\exp \left[-\frac{\pi^{2} r^{2} \alpha_{s}\left(\mu^{2}\right) x g\left(x, \mu^{2}\right)}{3 \sigma_{0}}\right]\right) .
$$

The evolution scale $\mu^{2}$ is connected to the size of the dipole by $\mu^{2}=C / r^{2}+\mu_{0}^{2}$. This assumption allows treating consistently the contributions of large $r$ without making the strongcoupling constant $\alpha_{s}\left(\mu^{2}\right)$ unphysically large.

The gluon density, which is parametrized at the starting scale $\mu_{0}^{2}$, is evolved to larger scales $\mu^{2}$ using leading order (LO) or next-to-LO (NLO) DGLAP evolution. For the initial condition, we consider here the soft ansatz as used in the original BGK model,

$$
x g\left(x, \mu_{0}^{2}\right)=A_{g} x^{-\lambda_{g}}(1-x)^{C_{g}} .
$$

The free parameters for this model are $\sigma_{0}, \mu_{0}^{2}$, and the parameters for gluon $A_{g}, \lambda_{g}$, and $C_{g}$. Their values have been obtained by a fit to the data using the XFITTER framework [35] in Refs. [36,37]. The fit results were found to be independent on the parameter $C$, which was therefore fixed as $C=4$, in agreement with the original BGK fits. For convenience, we show the parameters in Table I. Note that, for the BGK fit, that, although it has a fairly large error, the value of $\sigma_{0}$ is significantly larger than for, e.g., the GBW parametrization. The large $\sigma_{0}$ value of $\sim 90 \mathrm{mb}$ for the BGK dipole model was discussed previously in Ref. [37] where a comprehensive analysis of the newest HERA data from 2015 were performed. There it was observed that the values of the parameter $\sigma_{0}$ of a dipole cross section were also quite high on the order of $70 \mathrm{mb}$ for the fit with $Q_{0}^{2}=1.9 \mathrm{GeV}^{2}$ and $220 \mathrm{mb}$ for the fit with $Q_{0}^{2}=1.1 \mathrm{GeV}^{2}$. This is much higher than in the original GBW and BGK model fits where this number was around $23 \mathrm{mb}$. This is an interesting result because it indicates that the exponential form of the dipole cross section may be of little importance because, in the limit of very high values of $\sigma_{0}$, the dipole cross section reduces to the first term of the expansion of the exponent in the dipole cross section.

\section{IIM model}

Another parametrization of the dipole cross section which gives the latter in a simple analytic form is the IIM model [38]. It is also meant to take into account the saturation effects. Although the GBW and BGK models use for saturation the eikonal approximation, the IIM model uses a simplified version of the Balitsky-Kovchegov equation [29,30]. Here, we use a parametrization obtained by Soyez which includes heavy quarks into the fit [39]. The model was compared with the recent $\mathrm{H} 1$ Collaboration data in Ref. [40] where it was shown that it provides a good data description in the lower- $Q^{2}$ range of $0.2<Q^{2}<40 \mathrm{GeV}^{2}$. As this model also applies to the range of moderately large $Q^{2}$, it is an appropriate choice for our problem. The dipole cross section is parametrized as

$$
\sigma(x, r)=2 \pi R_{p}^{2} \begin{cases}N_{0} \exp \left[-2 \gamma L-\frac{L^{2}}{\kappa \lambda Y}\right], & \text { if } L \geqslant 0, \\ 1-\exp \left[-a\left(L-L_{0}\right)^{2}\right], & \text { else, }\end{cases}
$$

where

$$
L=\ln \left(\frac{2}{r Q_{s}}\right), \quad Q_{s}^{2}=\left(\frac{x_{0}}{x}\right)^{\lambda} \mathrm{GeV}^{2}, \quad Y=\ln \left(\frac{1}{x}\right) .
$$

and

$$
L_{0}=\frac{1-N_{0}}{\gamma N_{0}} \ln \left(\frac{1}{1-N_{0}}\right), \quad a=\frac{1}{L_{0}^{2}} \ln \left(\frac{1}{1-N_{0}}\right) .
$$

We take the numerical values found in the XFITTER code,

$$
\begin{aligned}
N_{0} & =0.7, \quad R_{p}=3.44 \mathrm{GeV}^{-1}, \quad \gamma=0.7376, \quad \kappa=9.9, \\
\lambda & =0.2197, \quad x_{0}=1.632 \times 10^{-5} .
\end{aligned}
$$

TABLE I. BGK fit with fitted valence quarks for $\sigma_{r}$ for H1ZEUS-NC data in the range of $Q^{2} \geqslant 3.5 \mathrm{GeV}^{2}$ and $x \leqslant 0.01$. NLO fit. Soft gluon. $m_{\text {uds }}=0.14, m_{c}=1.3 \mathrm{GeV} . Q_{0}^{2}=1.9 \mathrm{GeV}^{2}$.

\begin{tabular}{lccccrr}
\hline \hline$Q_{\min }^{2}\left(\mathrm{GeV}^{2}\right)$ & $\sigma_{0}(\mathrm{mb})$ & $A_{g}$ & $\lambda_{g}$ & $C_{g}$ & $N_{d f}$ & $\chi^{2}$ \\
\hline 3.5 & $89.99 \pm$ & $2.44 \pm$ & $-0.079 \pm$ & $7.24 \pm$ & 530 & 540.35 \\
& 9.2 & 0.145 & 0.099 & 0.61 & & \\
\hline \hline
\end{tabular}




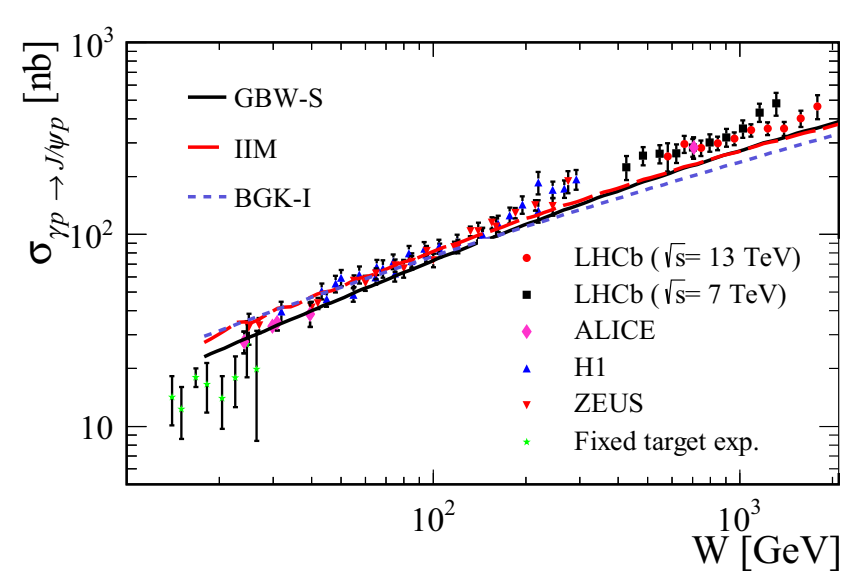

FIG. 2. Total cross section for the exclusive photoproduction $\gamma p \rightarrow J / \psi p$ as a function of $\gamma p$-c.m. system energy $W$. The data are from Refs. [41-49]. The results for three different dipole cross sections are shown.

\section{NUMERICAL RESULTS}

\section{A. Predictions for $J / \psi$ production on the proton target}

Let us now turn to the numerical results we obtained for the total exclusive photoproduction cross section of $J / \psi$ on the proton target. For the GBW and IIM dipole cross sections, we calculate the total cross section from

$$
\sigma(\gamma p \rightarrow J / \psi p ; W)=\frac{1+\rho_{N}^{2}}{16 \pi B} R_{\text {skewed }}^{2}|\langle V|\sigma(x, r)| \gamma\rangle|^{2} .
$$

The diffraction slope $B$ is taken as $B=B_{0}+4 \alpha^{\prime} \ln \left(W / W_{0}\right)$ with $B_{0}=4.88, \alpha^{\prime}=0.164 \mathrm{GeV}^{-2}$, and $W_{0}=90 \mathrm{GeV}$. We use the quark mass $m_{c}=1.4 \mathrm{GeV}$, except for the BGK fit where for consistency $m_{c}=1.3 \mathrm{GeV}$ was used. For the BGK type of parametrizations, it proves to be more stable numerically to substitute the "skewed glue" in the exponent,

$$
\sigma(x, r)=\sigma_{0}\left(1-\exp \left[-\frac{\pi^{2} r^{2} \alpha_{s}\left(\mu^{2}\right) R_{\text {skewed }} x g\left(x, \mu^{2}\right)}{3 \sigma_{0}}\right]\right),
$$

where the exponent $\Delta_{\text {IP }}$ which enters the Shuvaev factor is calculated from the relevant gluon distribution. This avoids taking tedious derivatives in the numerical grid for the dipole cross section. Our results are shown if Fig. 2 where we compare the results from the three different parametrizations of the dipole cross section against the data from Refs. [41-49]. We observe that the range of $30 \lesssim W \lesssim 300 \mathrm{GeV}$ is reasonably well described by all dipole cross sections. The very highenergy domain is covered by data which have been extracted from the $p p \rightarrow p p J / \psi$ reaction by the $\mathrm{LHCb}$ collaboration $[47,48]$. Here, none of the models do a good job. Although one could certainly try to obtain a dipole cross section which also fits the high-energy data, possibly by including vector meson data into the fit, this is not necessary for the purpose of this paper. Namely, it is the HERA energy range which will be most crucial for the calculations in ultraperipheral heavy-ion collisions later on. In that respect, the description
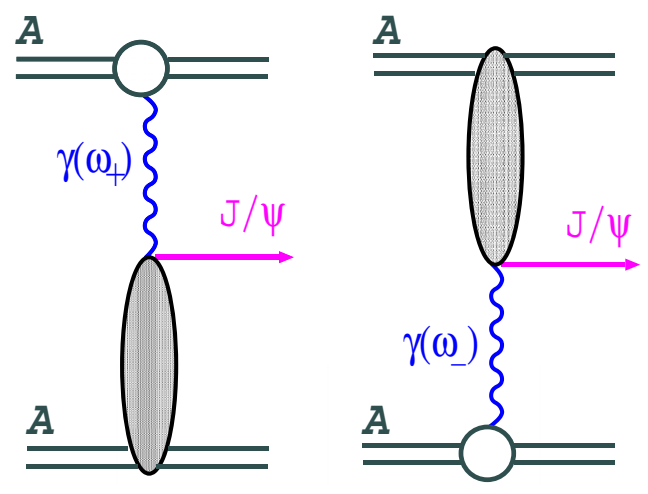

FIG. 3. Exclusive photoproduction in ultraperipheral heavy-ion collisions.

of free-nucleon data for the chosen dipole cross sections is satisfactory for our purposes.

\section{B. Results for photoproduction in ultraperipheral collisions}

We now turn to our results for ultraperipheral heavy-ion collisions. We obtain the rapidity-dependent cross section for exclusive $J / \psi$ production from the Weizsäcker-Williams fluxes of quasireal photons $n(\omega)$ as

$$
\begin{aligned}
& \frac{d \sigma\left(A A \rightarrow A A J / \psi ; \sqrt{s_{N N}}\right)}{d y} \\
& =n\left(\omega_{+}\right) \sigma\left(\gamma A \rightarrow J / \psi A ; W_{+}\right) \\
& \quad+n\left(\omega_{-}\right) \sigma\left(\gamma A \rightarrow J / \psi A ; W_{-}\right) .
\end{aligned}
$$

Here, the two terms correspond to the contributions where the left-moving ion serves as the photon source, and the right-moving one serves as the target and vice versa (see Fig. 3). Note that we neglected the interference between the two processes. This interference is concentrated at very small transverse momenta [8,50,51]. It introduces the azimuthal correlation between the outgoing ions, and in the absence of absorptive corrections, it would vanish after the angular integration [51].

We use the standard form of the Weizsäcker-Williams flux (see, e.g., the reviews [52,53]) for the ion moving with boost $\gamma$,

$$
n(\omega)=\frac{2 Z^{2} \alpha_{\mathrm{em}}}{\pi}\left[\xi K_{0}(\xi) K_{1}(\xi)-\frac{\xi^{2}}{2}\left[K_{1}^{2}(\xi)-K_{0}^{2}(\xi)\right]\right] .
$$

Here, $\omega$ is the photon energy, and $\xi=2 R_{A} \omega / \gamma$. This flux was obtained by imposing the constraint on the impact parameter of the collision $b>2 R_{A}$ where we use $R_{A}=7 \mathrm{fm}$. This means that configurations where nuclei touch each other are excluded as otherwise inelastic processes would destroy the rapidity gaps in the event. The photon energies corresponding to the two contributions are $\omega_{ \pm}=m_{V} \exp [ \pm y] / 2$, the corresponding c.m. system energies for the $\gamma A \rightarrow J / \psi A$ subprocesses are $W_{ \pm}^{2}=2 \sqrt{s_{N N}} \omega_{ \pm}$.

In order to understand the kinematics a bit better, in Tables II and III, we show the values of $W_{ \pm}$, the associated Bjorken- $x$ values $x_{ \pm}$, as well as photon fluxes $n\left(\omega_{ \pm}\right)$. For 
TABLE II. Subenergies $W_{ \pm}$and Bjorken- $x$ values $x_{ \pm}$for $\sqrt{s_{N N}}=2.76 \mathrm{TeV}$ for a given rapidity $y$. Also shown are photon fluxes $n\left(\omega_{ \pm}\right)$and the photoproduction cross sections on ${ }^{208} \mathrm{~Pb}$ at energies $W_{ \pm}$for the IIM-dipole cross section.

\begin{tabular}{|c|c|c|c|c|c|c|c|c|}
\hline $\begin{array}{l}\sqrt{s_{N N}}=2.76 \mathrm{TeV} \\
y\end{array}$ & $W_{+}(\mathrm{GeV})$ & $W_{-}(\mathrm{GeV})$ & $x_{+}$ & $x_{-}$ & $n\left(\omega_{+}\right)$ & $n\left(\omega_{-}\right)$ & $\sigma\left(W_{+}\right)(\mu \mathrm{b})$ & $\sigma\left(W_{-}\right)(\mu \mathrm{b})$ \\
\hline 0.0 & 92.5 & 92.5 & $1.12 \times 10^{-3}$ & $1.12 \times 10^{-3}$ & 69.4 & 69.4 & 27.4 & 27.4 \\
\hline 2.0 & 251 & 34.0 & $1.52 \times 10^{-4}$ & $8.29 \times 10^{-3}$ & 14.5 & 132 & 48.6 & 13.0 \\
\hline 3.0 & 414 & 20.6 & $5.59 \times 10^{-5}$ & $2.25 \times 10^{-2}$ & 1.68 & 163 & 62.2 & 7.18 \\
\hline 3.8 & 618 & 13.8 & $2.51 \times 10^{-5}$ & $5.02 \times 10^{-2}$ & 0.03 & 188 & 74.6 & 2.81 \\
\hline
\end{tabular}

convenience we have also included the values of the photoproduction cross-section $\sigma\left(\gamma A \rightarrow J / \psi A: W_{ \pm}\right)$for the example of the IIM dipole cross section and for the ${ }^{208} \mathrm{~Pb}$ nucleus. Table II is for the energy $\sqrt{s_{N N}}=2.76 \mathrm{TeV}$, and Table III is for $\sqrt{s_{N N}}=5.02 \mathrm{TeV}$. At midrapidity of course $W_{ \pm}$coincide, and we are always well in the energy range that has been explored at HERA for the free-nucleon target. If we move out to larger rapidities of the two processes, it is the low-energy reaction, which tends to dominate. This has its explanation in the rather quick drop of the nuclear photon fluxes at high photon energies and the modest rise of the nuclear photoproduction cross section.

In Fig. 4, we show the cross section as a function of $J / \psi$ rapidity for $\sqrt{s_{N N}}=2.76 \mathrm{TeV}$ for the three different dipole cross sections introduced previously. We compare them to the data obtained by the ALICE [2,3] and CMS [4] Collaborations. We see that at large rapidities we obtain a fair description of the data, whereas all of the dipole cross sections overestimate the data at midrapidity.

In Fig. 5, the rapidity-dependent cross section is shown at $\sqrt{s_{N N}}=5.02 \mathrm{TeV}$ and compared to preliminary data from the ALICE Collaboration [5]. Figure 6 also is calculated at $\sqrt{s_{N N}}=5.02 \mathrm{TeV}$ but compares to the preliminary data by the $\mathrm{LHCb}$ Collaboration [6]. We see that again we get a reasonable description of the preliminary data. We should point out that we did not include a skewedness correction with our results on nuclear targets. It is not completely clear how the skewedness correction should be applied in the case of nuclear rescatterings. Obviously the assumption of a two-gluon exchange does not apply to our mechanism, and the necessary longitudinal momentum transfer can be shared by many gluons. As the diffractive amplitude has a "formfactor"-like (perhaps exponential) behavior an equipartition of longitudinal momentum transfers looks more likely than having one large momentum transfer and many small ones. In addition, the data at large rapidities have a large contribution from not very small $x$ where the skewedness correction may not be justified. We therefore follow the authors of Ref. [15] and omit the skewedness correction.

The overall picture suggests that the Glauber-Gribov formalism in the color dipole approach works reasonably well at not too high energies (or not too small $x$ ), whereas at higher energies ( $\operatorname{smaller} x$ ), there is room for additional nuclear suppression.

This point is also borne out by Fig. 7 where we show directly the total cross section for diffractive photoproduction of $J / \psi$ on ${ }^{208} \mathrm{~Pb}$. The data points shown have been extracted by Contreras [54]. A good description of Contreras' data has been previously achieved in a phenomenological model based on a geometric scaling ansatz [55].

We see that at high energies we overestimate the data. However even at the highest energy $W \approx 470 \mathrm{GeV}$, partially due to the large error bar, the required correction is not very large.

In fact, in our calculations, we included only the rescattering of the $c \bar{c}$ pair. Due to the "scanning-radius" property of vector meson photoproduction, in rescatterings of the small $c \bar{c}$ pair are, in fact, higher twist effects. This is different than the case of the inclusive structure function $F_{2}$ where large dipoles contribute to a scaling (up to logarithms) nuclear shadowing [28].

It is well understood that at small $x$, the coherency condition can be also satisfied by higher Fock $c \bar{c} g, c \bar{c} g g \cdots$ states. In fact, these Fock states (in a configuration of strongly ordered transverse sizes) are responsible for the DGLAP evolution of structure functions, whereas configurations strongly ordered in gluon longitudinal momenta correspond to the Balitsky-Fadin-Kuraev-Lipatov-BK (BFKL-BK) limit [56].

TABLE III. Subenergies $W_{ \pm}$and Bjorken- $x$ values $x_{ \pm}$for $\sqrt{s_{N N}}=5.02 \mathrm{TeV}$ for a given rapidity $y$. Also shown are photon fluxes $n\left(\omega_{ \pm}\right)$ and the photoproduction cross sections on ${ }^{208} \mathrm{~Pb}$ at energies $W_{ \pm}$for the IIM-dipole cross section.

\begin{tabular}{|c|c|c|c|c|c|c|c|c|}
\hline $\begin{array}{l}\sqrt{s_{N N}}=5.02 \mathrm{TeV} \\
y\end{array}$ & $W_{+}(\mathrm{GeV})$ & $W_{-}(\mathrm{GeV})$ & $x_{+}$ & $x_{-}$ & $n\left(\omega_{+}\right)$ & $n\left(\omega_{-}\right)$ & $\sigma\left(W_{+}\right)(\mu \mathrm{b})$ & $\sigma\left(W_{-}\right)(\mu \mathrm{b})$ \\
\hline 0.0 & 125 & 125 & $6.17 \times 10^{-4}$ & $6.17 \times 10^{-4}$ & 87.9 & 87.9 & 32.9 & 32.9 \\
\hline 2.0 & 339 & 45.9 & $8.35 \times 10^{-5}$ & $4.56 \times 10^{-3}$ & 28.5 & 150 & 56.5 & 16.8 \\
\hline 3.0 & 559 & 27.8 & $3.07 \times 10^{-5}$ & $1.24 \times 10^{-2}$ & 7.5 & 181 & 71.3 & 10.6 \\
\hline 4.0 & 921 & 16.9 & $1.13 \times 10^{-5}$ & $3.37 \times 10^{-2}$ & 0.35 & 213 & 88.6 & 4.98 \\
\hline
\end{tabular}




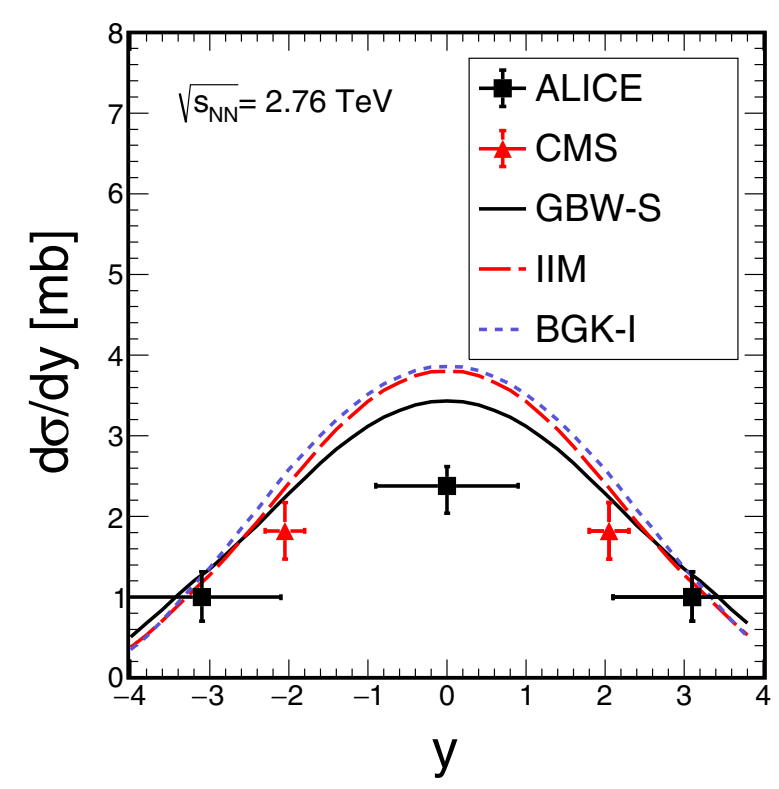

FIG. 4. Rapidity-dependent cross-section $d \sigma / d y$ for exclusive production of $J / \psi$ in ${ }^{208} \mathrm{~Pb}^{208} \mathrm{~Pb}$ collisions at per-nucleon c.m. system energy $\sqrt{s_{N N}}=2.76 \mathrm{TeV}$. The data are from the ALICE $[2,3]$ and CMS [4] Collaborations.

We believe that the missing higher Fock states are the main culprit behind the overprediction of the ALICE Collaboration data at midrapidity. Whether their effect can eventually be absorbed into a leading-twist shadowing correction to the DGLAP evolving nuclear glue is an open issue at the moment. Some attempts to include gluon shadowing by renormalizing the dipole cross section have been made in Ref. [57], following Ref. [58].

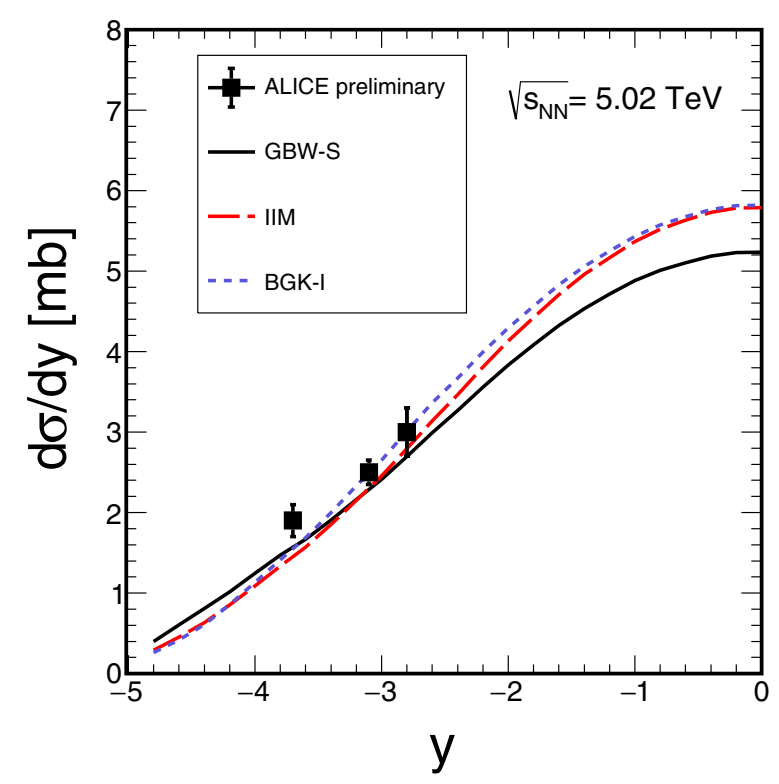

FIG. 5. Rapidity-dependent cross-section $d \sigma / d y$ for exclusive production of $J / \psi$ in ${ }^{208} \mathrm{~Pb}{ }^{208} \mathrm{~Pb}$ collisions at per-nucleon c.m. system energy $\sqrt{s_{N N}}=5.02 \mathrm{TeV}$. Shown are preliminary data are from the ALICE Collaboration [5].

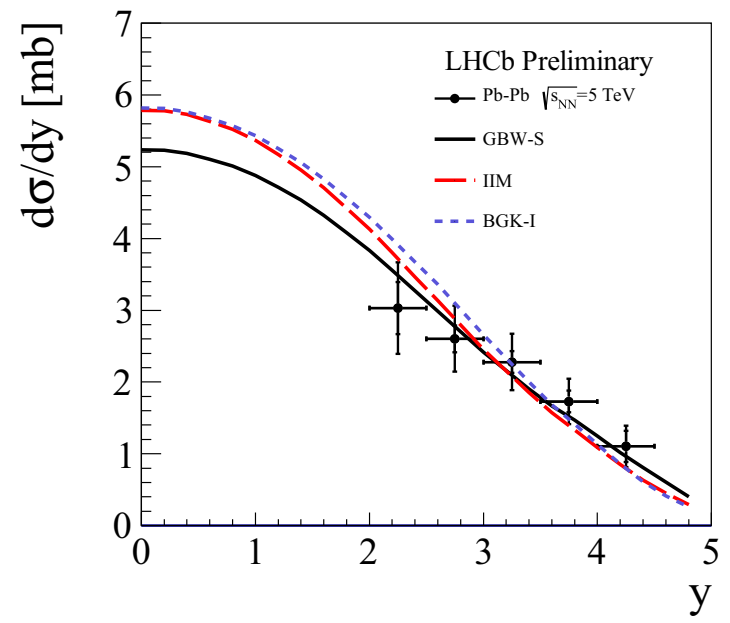

FIG. 6. Rapidity-dependent cross-section $d \sigma / d y$ for exclusive production of $J / \psi$ in ${ }^{208} \mathrm{~Pb}^{208} \mathrm{~Pb}$ collisions at per-nucleon c.m. system energy $\sqrt{s_{N N}}=5.02 \mathrm{TeV}$. Shown are preliminary data are from the LHCb Collaboration [6].

One may ask finally if the light-cone wave function can be the scapegoat. Indeed, in a careful analysis of some theoretical uncertainties [14] (see also, e.g., Ref. [17]), it was shown that there can be a sizable dependence on the meson light-cone wave function. However, here we take the point of view that the successful description of free-nucleon HERA data in the energy range fix the wave-function overlap. Indeed, with the dipole cross sections we employed, we would rather spoil the agreement with HERA data if we substitute another of the popular parametrizations. From this point of view, attempts to obtain light-cone wave functions from other sources [59] are interesting.

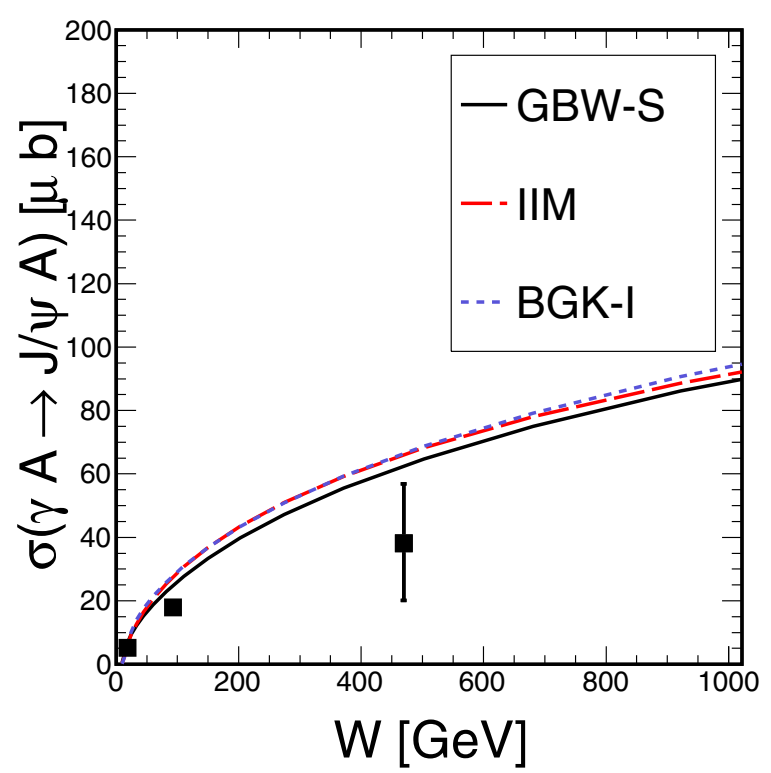

FIG. 7. The total cross-section $\sigma(\gamma A \rightarrow J / \psi A)$ for the ${ }^{208} \mathrm{~Pb}$ nucleus as a function of $\gamma A$-c.m. energy $W$. The data are from Ref. [54]. 
A different approach has recently been taken in Ref. [60], where a reasonable agreement with ALICE Collaboration data has been obtained by modifying the nuclear impact parameter averaging of dipole amplitudes. The "hot-spot" model that does a reasonable job against data differs from the standard picture of Glauber theory preferred by us. We believe that the Glauber approach is theoretically well motivated and should be taken as the starting point.

\section{SUMMARY AND OUTLOOK}

In this paper, we have presented calculations using the Glauber-Gribov theory for coherent exclusive photoproduction of $J / \psi$ mesons on heavy nuclei within the color dipole approach. The dipole cross sections which we utilize have all been obtained from fitting inclusive deep-inelastic structure function data from HERA. We first calculated the total elastic photoproduction of $J / \psi$ on the free nucleon comparing to the data available from fixed-target experiments from the $\mathrm{H} 1$ and ZEUS Collaborations at HERA as well as to data extracted from $p p$ or $p A$ collisions by the LHCb and ALICE Collaborations. All the three-dipole cross sections used in this paper give a reasonable description of the data, up to and including the HERA energy range, when used together with the so-called boosted Gaussian parametrization of the $J / \psi$ wave function. The higher-energy data extracted mainly by the LHCb Collaboration from exclusive $p p$ collisions are not well described.

We have applied our results to the exclusive $J / \psi$ production in heavy-ion (lead-lead) collisions at the energies $\sqrt{s_{N N}}=2.76$ and $\sqrt{s_{N N}}=5.02 \mathrm{GeV}$.
The color dipoles play the role of the eigenstates of the scattering matrix and take into account the inelastic shadowing corrections. We have taken into account the rescattering of a $c \bar{c}$ dipole in the nucleus including the real part of the free-nucleon amplitude consistent with the rules of Glauber theory.

Although there is substantial uncertainty as to how to include the skewedness correction in to the nuclear amplitude, the description of published and preliminary data can be regarded satisfactory. However, the data point taken by the ALICE Collaboration at midrapidity for $\sqrt{s_{N N}}=2.76 \mathrm{TeV}$ is overpredicted. This seems to point to the fact that rescattering of the $c \bar{c}$ dipole is insufficient at energies $W_{\gamma A} \sim 100 \mathrm{GeV}$ or $x \approx 0.001$.

We believe that explicit account of higher Fock states is necessary in this kinematic region. This is consistent with an analysis of nuclear shadowing and deep inelastic diffraction on nuclei in Ref. [61]. There it is shown that $q \bar{q} g$ states are important for nuclear shadowing at $x \lesssim 0.005$.

Whether the correct approach is a resummation of their effect in a BFKL-BK framework, or whether they can be absorbed into a leading-twist shadowing of the collinear nuclear glue is an open issue. It stands to reason that this issue can hardly be resolved by only one observable, measured essentially at one hard scale, and that future measurements at an electron-ion collider will be crucial for a deeper understanding of the nuclear glue.

\section{ACKNOWLEDGMENTS}

This work was partially supported by the Polish National Science Center Grant No. DEC-2014/15/B/ST2/02528.
[1] S. R. Klein and J. Nystrand, Phys. Rev. C 60, 014903 (1999).

[2] ALICE Collaboration, B. Abelev et al., Phys. Lett. B 718, 1273 (2013).

[3] ALICE Collaboration, E. Abbas et al., Eur. Phys. J. C 73, 2617 (2013).

[4] CMS Collaboration, V. Khachatryan et al., Phys. Lett. B 772, 489 (2017).

[5] ALICE Collaboration, E. L. Kryshen, Nucl. Phys. A 967, 273 (2017).

[6] LHCb Collaboration, A. Bursche, Nucl. Phys. A 982, 247 (2019).

[7] J. G. Contreras and J. D. Tapia Takaki, Int. J. Mod. Phys. A 30, 1542012 (2015).

[8] S. R. Klein and J. Nystrand, Phys. Rev. Lett. 92, 142003 (2004).

[9] V. P. Gonçalves and M. V. T. Machado, Eur. Phys. J. C 40, 519 (2005).

[10] A. L. Ayala Filho, V. P. Gonçalves, and M. T. Griep, Phys. Rev. C 78, 044904 (2008).

[11] A. Cisek, W. Schäfer, and A. Szczurek, Phys. Rev. C 86, 014905 (2012).

[12] T. Lappi and H. Mäntysaari, Phys. Rev. C 87, 032201(R) (2013).
[13] V. Guzey, E. Kryshen, M. Strikman, and M. Zhalov, Phys. Lett. B 726, 290 (2013).

[14] G. Sampaio dos Santos and M. V. T. Machado, J. Phys. G 42, 105001 (2015).

[15] V. P. Gonçalves, M. V. T. Machado, B. D. Moreira, F. S. Navarra, and G. S. dos Santos, Phys. Rev. D 96, 094027 (2017).

[16] V. Guzey, E. Kryshen, and M. Zhalov, Phys. Rev. C 93, 055206 (2016).

[17] Y.-p. Xie and X. Chen, Eur. Phys. J. C 76, 316 (2016).

[18] F. Kopp and M. V. T. Machado, Phys. Rev. D 98, 014010 (2018).

[19] I. P. Ivanov, N. N. Nikolaev, and A. A. Savin, Phys. Part. Nucl. 37, 1 (2006).

[20] A. Łuszczak and W. Schäfer, Phys. Rev. C 97, 024903 (2018).

[21] N. N. Nikolaev, Comments Nucl. Part. Phys. 21, 41 (1992).

[22] J. Nemchik, N. N. Nikolaev, and B. G. Zakharov, Phys. Lett. B 341, 228 (1994).

[23] J. Nemchik, N. N. Nikolaev, E. Predazzi, and B. G. Zakharov, Z. Phys. C 75, 71 (1997).

[24] H. Kowalski, L. Motyka, and G. Watt, Phys. Rev. D 74, 074016 (2006).

[25] A. G. Shuvaev, K. J. Golec-Biernat, A. D. Martin, and M. G. Ryskin, Phys. Rev. D 60, 014015 (1999). 
[26] R. J. Glauber, Lectures in Theoretical Physics, edited by W. E. Brittin and L. J. Dunham (Interscience, New York, 1959), Vol. 1, pp. 315-414.

[27] E. S. Abers, H. Burkhardt, V. L. Teplitz, and C. Wilkin, Nuovo Cimento A 42, 365 (1966); J. Pumplin and M. Ross, Phys. Rev. Lett. 21, 1778 (1968); V. N. Gribov, Zh. Eksp. Teor. Fiz. 56, 892 (1969) [Sov. Phys. JETP 29, 483 (1969)]; 57, 1306 (1969) [30, 709 (1970)].

[28] N. N. Nikolaev and B. G. Zakharov, Z. Phys. C 49, 607 (1991).

[29] I. Balitsky, Nucl. Phys. B 463, 99 (1996).

[30] Y. V. Kovchegov, Phys. Rev. D 60, 034008 (1999).

[31] K. J. Golec-Biernat and M. Wüsthoff, Phys. Rev. D 59, 014017 (1998).

[32] K. J. Golec-Biernat and M. Wusthoff, Phys. Rev. D 60, 114023 (1999).

[33] K. Golec-Biernat and S. Sapeta, J. High Energy Phys. 03 (2018) 102.

[34] J. Bartels, K. J. Golec-Biernat, and H. Kowalski, Phys. Rev. D 66, 014001 (2002).

[35] S. Alekhin et al., Eur. Phys. J. C 75, 304 (2015); H1 and ZEUS Collaborations, H. Abramowicz et al., ibid. 75, 580 (2015); M. Botje, Comput. Phys. Commun. 182, 490 (2011); F. James and M. Roos, ibid. 10, 343 (1975); R. S. Thorne and R. G. Roberts, Phys. Rev. D 57, 6871 (1998); R. S. Thorne, ibid. 73, 054019 (2006); H1 Collaboration, F. D. Aaron et al., Eur. Phys. J. C 63, 625 (2009); ZEUS Collaboration, S. Chekanov et al., ibid. 42, 1 (2005); J. Pumplin, D. R. Stump, and W. K. Tung, Phys. Rev. D 65, 014011 (2001); J. Pumplin, D. R. Stump, J. Huston, H. L. Lai, P. M. Nadolsky, and W. K. Tung, J. High Energy Phys. 07 (2002) 012.

[36] A. Łuszczak and H. Kowalski, Phys. Rev. D 89, 074051 (2014).

[37] A. Łuszczak and H. Kowalski, Phys. Rev. D 95, 014030 (2017).

[38] E. Iancu, K. Itakura, and S. Munier, Phys. Lett. B 590, 199 (2004).

[39] G. Soyez, Phys. Lett. B 655, 32 (2007).

[40] H1 Collaboration, F. D. Aaron et al., Eur. Phys. J. C 71, 1579 (2011).

[41] M. E. Binkley et al., Phys. Rev. Lett. 48, 73 (1982).
[42] B. H. Denby et al., Phys. Rev. Lett. 52, 795 (1984).

[43] E687 Collaboration, P. L. Frabetti et al., Phys. Lett. B 316, 197 (1993).

[44] H1 Collaboration, C. Alexa et al., Eur. Phys. J. C 73, 2466 (2013).

[45] H1 Collaboration, A. Aktas et al., Eur. Phys. J. C 46, 585 (2006).

[46] ZEUS Collaboration, S. Chekanov et al., Eur. Phys. J. C 24, 345 (2002).

[47] LHCb Collaboration, R. Aaij et al., J. Phys. G: Nucl. Part. Phys. 41, 055002 (2014).

[48] LHCb Collaboration, R. Aaij et al., J. High Energy Phys. 10 (2018) 167.

[49] ALICE Collaboration, B. B. Abelev et al., Phys. Rev. Lett. 113, 232504 (2014).

[50] K. Hencken, G. Baur, and D. Trautmann, Phys. Rev. Lett. 96, 012303 (2006).

[51] W. Schäfer and A. Szczurek, Phys. Rev. D 76, 094014 (2007).

[52] C. A. Bertulani, S. R. Klein, and J. Nystrand, Annu. Rev. Nucl. Part. Sci. 55, 271 (2005).

[53] G. Baur, K. Hencken, D. Trautmann, S. Sadovsky, and Y. Kharlov, Phys. Rep. 364, 359 (2002).

[54] J. G. Contreras, Phys. Rev. C 96, 015203 (2017).

[55] F. G. Ben, M. V. T. Machado, and W. K. Sauter, Phys. Rev. D 96, 054015 (2017).

[56] N. N. Nikolaev and B. G. Zakharov, Z. Phys. C 64, 631 (1994).

[57] M. B. Gay Ducati, M. T. Griep, and M. V. T. Machado, Phys. Rev. C 88, 014910 (2013).

[58] Y. P. Ivanov, B. Z. Kopeliovich, A. V. Tarasov, and J. Hufner, Phys. Rev. C 66, 024903 (2002).

[59] G. Chen, Y. Li, P. Maris, K. Tuchin, and J. P. Vary, Phys. Lett. B 769, 477 (2017).

[60] J. Cepila, J. G. Contreras, and M. Krelina, Phys. Rev. C 97, 024901 (2018).

[61] N. N. Nikolaev, W. Schäfer, B. G. Zakharov, and V. R. Zoller, JETP Lett. 84, 537 (2007). 